Research Article

Open Access

Fateme Ghomanjani* and Stanford Shateyi

\title{
A new approach for solving Bratu's problem
}

https://doi.org/10.1515/dema-2019-0023

Received December 23, 2018; accepted July 21, 2019

Abstract: A numerical technique for one-dimensional Bratu's problem is displayed in this work. The technique depends on Bernstein polynomial approximation. Numerical examples are exhibited to verify the efficiency and accuracy of the proposed technique. In this sequel, the obtained error was shown between the proposed technique, Chebyshev wavelets, and Legendre wavelets. The results display that this technique is accurate.

Keywords: Bernstein polynomials and series, numerical solution, Bezier method

MSC: 34B10, 34B15

\section{Introduction}

In this sequel, Liouville-Bratu-Gelfand equation is considered (see [1])

$$
\begin{aligned}
& \Delta u+\lambda e^{u}=0, \quad x \in \Omega, \\
& u=0, \quad x \in \partial \Omega,
\end{aligned}
$$

where $\lambda>0$ is a physical parameter and $\Omega \in R^{N}$ is a bounded domain.

Now, one may focus on the boundary value problem and initial value problem of Bratu's problem. Here, the Bratu's boundary value problem in one-dimensional planner coordinates is as follows:

$$
\begin{aligned}
& u^{\prime \prime}(x)+\lambda e^{u(x)}=0, \quad 0<x<1, \\
& u(0)=u(1)=0, \quad \lambda>0,
\end{aligned}
$$

where the exact solution of this problem is (see [2])

$$
\begin{aligned}
& u(x)=-2 \ln \left[\frac{\cosh (0.5 \theta(x-0.5))}{\cosh (0.25 \theta)}\right], \\
& \theta=\sqrt{2 \lambda} \sinh (0.25 \theta) .
\end{aligned}
$$

The problem has zeros when $\lambda>\lambda_{c}, \lambda=\lambda_{c}$ and $\lambda<\lambda_{c}$, separately, where the critical value $\lambda_{c}$ satisfies the following equation

$$
1=\frac{1}{4} \sqrt{2 \lambda_{c}} \cosh \left(\frac{1}{4} \theta\right) .
$$

The critical value $\lambda_{c}$ was calculated in [2-4] and found to be $\lambda_{c}=3.513830719$.

This solution displays a bifurcation pattern, which only characterizes nonlinear differential equations. In fact, the following one-dimensional Bratu's problem is utilized in various types of applications such as

\footnotetext{
*Corresponding Author: Fateme Ghomanjani: Department of Mathematics, Kashmar Higher Education Institute, Kashmar, Iran; E-mail: fatemeghomanjani@gmail.com, f.ghomanjani@kashmar.ac.ir Stanford Shateyi: Department of Mathematics, University of Venda, P Bag X5050, Thohoyandou 0950, South Africa; E-mail: stanford.shateyi@univen.ac.za
} 
the fuel ignition model of the thermal combustion theory, radioactive heat transfer and nanotechnology (see [5-20]). In this paper, we have the following Bratu's boundary value problem:

$$
\begin{aligned}
& u^{\prime \prime}(x)+\lambda e^{u(x)}=0, \quad 0<x<1, \\
& u(0)=u^{\prime}(0)=0 .
\end{aligned}
$$

The way to solve a boundary or initial value problem consists of computing first the general solution to the differential equation and secondly of finding the arbitrary parameters by applying the boundary or initial conditions (see [21]). Boyd [3, 22] utilized Chebyshev polynomial expansions and the Gegenbauer polynomial expansion as base functions. Syam and Hamdan [6] exhibited the Laplace decomposition method for solving Bratu's problem. Additionally, Aksoy and Pakdemirli [23] improved a perturbation solution to Bratu's problem. Wazwaz [8] exhibited the Adomian decomposition method for solving Bratu's problem. Also, the uses of spline strategy, wavelet technique and Sinc-Galerkin method for solving the Bratu's problem have been utilized in [24, 25]. By utilizing the authors solved the Bratu's problem [26]. Additionally there are many papers and books that manage the Bezier curves. Harada, and Nakamae [27], Nürnberger and Zeilfelder [28] utilized the Bezier control points in approximating functions. Zheng et al. [29] proposed the utilization of control points of the Bezier curve for solving differential equations numerically. Also, to solve delay differential equations, the Bezier control points strategy is utilized in [30]. Some other uses of the Bezier functions are found in [31]. In the present work, we recommend a system like the one which was utilized in [31] for solving Bratu's problem. The use of Bezier curves for solving Bratu's problem is a novel idea. The approach used in this article reduces the CPU time.

This study is arranged as follows: In Section 2, the statement of the problem is presented. Error estimation is given in Section 3. Some numerical examples are provided in Section 4. Finally, Section 5 will give a brief conclusion.

\section{Statement of the problem}

The goal is to obtain an approximate solution of Eq.(2), presented in the following form:

$$
u(x) \cong u_{n+1}(x)=\sum_{i=0}^{n} c_{i} B_{i, n}(x), 0 \leq x \leq 1, n \geq 1,
$$

where $\left\{B_{i, n}(x)\right\}_{i=0}^{n}$ denotes the Bernstein polynomials (B-polynomials) of $n$-th degree, as introduced by

$$
\begin{aligned}
& B_{i, n}\left(\frac{x-x_{0}}{h}\right)=\left(\begin{array}{c}
n \\
i
\end{array}\right) \frac{1}{h^{n}}\left(x_{f}-x\right)^{n-i}\left(x-x_{0}\right)^{i}, \\
& 0=x_{0} \leq x \leq x_{f}=1, \quad i=0,1, \ldots, n, h=x_{f}-x_{0},
\end{aligned}
$$

where the unknown control points are $c_{i}, i=0,1, \ldots, n$.

In this paper, the Bezier curve is utilized for solving the Bratu's problem. This technique is applied in [32, 33] for solving optimal control of switched systems and some linear optimal control systems with pantograph delays. The convergence of the proposed method was proven when $n$ tends to infinity $[32,33]$.

By substituting $u(x)$ in Eq. (2), we may characterize $f_{\text {objective }}$ for $x \in\left[x_{0}, x_{f}\right]$ as follows:

$$
f_{\text {objective }}=\sum_{i=0}^{n} c_{i}^{2} \text {. }
$$

Our goal is to solve the following problem to find the values $c_{r}$, for $r=0,1, \ldots, n$.

$$
\begin{array}{cl}
\text { Min } & f_{\text {objective }} \\
\text { such that } & u^{\prime \prime}(x)+\lambda e^{u}=0,0<x<1,
\end{array}
$$




$$
u(0)=u^{\prime}(0)=0
$$

where the first derivative and the second derivative of the Bezier curve are as follows:

$$
\begin{aligned}
& u^{\prime}(x) \cong n \sum_{i=0}^{n-1} B_{i, n}(x)\left(c_{i+1}-c_{i}\right), \\
& u^{\prime \prime}(x) \cong n(n-1) \sum_{i=0}^{n-2} B_{i, n-2}(x)\left(c_{i+2}-2 c_{i+1}+c_{i}\right) .
\end{aligned}
$$

Now, one may utilize lagrange multipliers technique for solving (8).

\section{Error estimation}

We may define

$$
\begin{aligned}
& (D u)(x)=u^{\prime \prime}(x)+\lambda e^{u(x)}=0, \\
& e^{u(x)}=1+u(x)+\frac{(u(x))^{2}}{2 !}+\frac{(u(x))^{3}}{3 !}+\ldots
\end{aligned}
$$

then

$$
\left(D u_{n}\right)(x)=u_{n}^{\prime \prime}(x)+\lambda e^{u_{n}(x)}=0,
$$

and by utilizing Eqs. (6), (9), we have

$$
\begin{aligned}
\left(D u_{n}\right)(x)= & n(n-1) \sum_{i=0}^{n-2} B_{i, n-2}(x)\left(c_{i+2}-2 c_{i+1}+c_{i}\right) \\
& +\lambda\left(1+\sum_{i=0}^{n} c_{i} B_{i, n}(x)+\frac{\left(\sum_{i=0}^{n} c_{i} B_{i, n}(x)\right)^{2}}{2 !}+\frac{\left(\sum_{i=0}^{n} c_{i} B_{i, n}(x)\right)^{3}}{3 !}+\ldots\right)=0 .
\end{aligned}
$$

Now, the following function is defined as

$$
\begin{array}{cl}
\min J= & J(C)=J\left(c_{0}, c_{1}, \ldots, c_{n}\right)=f_{\text {objective }}=\sum_{i=0}^{n} c_{i}^{2} \\
\text { such that } & \left(D u_{n}\right)(x)=u_{n}^{\prime \prime}(x)+\lambda e^{u_{n}(x)}=0, u_{n}(0)=u_{n}^{\prime}(0)=0 .
\end{array}
$$

We therefore conclude that an error estimation of the proposed method is represented.

Now, the approximate solution obtained by the proposed approach can be represented in the form of

$$
u_{n}(x)=\sum_{i=0}^{n} c_{i} B_{i, n}(x)
$$

Also, an upper bound for the error estimation of the proposed numerical scheme can be established in the following theorem.

Theorem 1. Suppose that $u(x) \in C^{n+1}[0,1]$ is the exact solution of this problem, $u_{n}(x)=\sum_{i=0}^{n} c_{i} B_{i, n}(x)$ is the truncated series solution with degree $n$. It holds that

$$
\begin{aligned}
& \left\|u(x)-u_{n}(x)\right\|_{\infty} \leq \frac{M}{(n+1) !} \max _{i=0,1, \ldots, n}\left|\tilde{c}_{i}\right|, \\
& M=\max _{0 \leq x \leq 1}\left|u^{n+1)}(x)\right|, \tilde{c_{k}}=\frac{u^{(k)}(0)}{k !}-c_{i} .
\end{aligned}
$$

Proof. See [34]. 
Now, the following problem is considered:

$$
\begin{aligned}
L\left(u(x), \frac{d u(x)}{d x}\right) & =\frac{d^{2} u(x)}{d x}+\lambda\left(1+u(x)+\frac{u^{2}(x)}{2 !}+\ldots\right) \\
& =F(x) \\
u(0) & =a \\
u^{\prime}(0) & =b .
\end{aligned}
$$

Lemma 1. For a polynomial in Bezier form

$$
u(x)=\sum_{i=0}^{n_{1}} c_{i, n_{1}} B_{i, n_{1}}(x),
$$

we have

$$
\frac{\sum_{i=0}^{n_{1}} c_{i, n_{1}}^{2}}{n_{1}+1} \geq \frac{\sum_{i=0}^{n_{1}+1} c_{i, n_{1}+1}^{2}}{n_{1}+2} \geq \ldots \geq \frac{\sum_{i=0}^{n_{1}+m_{1}} c_{i, n_{1}+m_{1}}^{2}}{n_{1}+m_{1}+1}
$$

where $c_{i, n_{1}+m_{1}}$ are the Bezier coefficients of $u(x)$ after it is degree-elevated to degree $n_{1}+m_{1}$.

Proof. See [29].

Theorem 2. If problem (14) has a unique $C^{2}[0,1]$ continuous solution $\bar{u}$, then the approximate solution obtained by the control-point-based method converges to the exact solution $\bar{u}$ as the degree of the approximate solution tends to infinity.

Proof. Given an arbitrary small positive number $\epsilon>0$, by the Weierstrass Theorem, one can easily find polynomials $Q_{1, N_{1}}(x)$ of degree $N_{1}$ such that

$$
\left\|Q_{1, N_{1}}(x)-\bar{u}(x)\right\|_{\infty} \leq \frac{\epsilon}{16 \times 2^{n}|\lambda|\left(\left\|Q_{1, N_{1}}(x)\right\|_{\infty}+\|\bar{u}(x)\|_{\infty}\right)},
$$

and

$$
\left\|\frac{d^{i} Q_{1, N_{1}}(x)}{d x^{i}}-\frac{d^{i} \bar{u}(x)}{d x^{i}}\right\|_{\infty} \leq \frac{\epsilon}{16 \times 2^{n}|\lambda|}, \quad i=1,2,
$$

where $\|\cdot\|_{\infty}$ stands for the $L_{\infty}$-norm over $[0,1]$. In particular, we have

$$
\left\|a-Q_{1, N_{1}}(0)\right\|_{\infty} \leq \frac{\epsilon}{16 \times 2^{n}|\lambda|} .
$$

In general, $Q_{1, N_{1}}(x)$ does not satisfies the boundary conditions. After a small perturbation with linear and constant polynomials $\alpha x+\beta$ for $Q_{1, N_{1}}(x)$, we can obtain polynomials $P_{1, N_{1}}(x)=Q_{1, N_{1}}(x)+(\alpha x+\beta)$ such that $P_{1, N_{1}}(x)$ satisfy the following boundary conditions

$$
P_{1, N_{1}}(0)=a,\left.\frac{d P_{1, N_{1}}(x)}{d x}\right|_{x=0}=b .
$$

Therefore, we have

$$
Q_{1, N_{1}}(0)+\beta=a,
$$

and

$$
\left.\frac{d Q_{1, N_{1}}(x)}{d x}\right|_{x=0}+\alpha=b
$$


By using (15), we have

$$
\begin{aligned}
& \left\|b-\left.\frac{d Q_{1, N_{1}}(x)}{d x}\right|_{\chi=0}\right\|_{\infty}=\|\alpha\|_{\infty} \leq \frac{\epsilon}{16 \times 2^{n}|\lambda|}, \\
& \left\|a-Q_{1, N_{1}}(0)\right\|_{\infty}=\|\beta\|_{\infty} \leq \frac{\epsilon}{16 \times 2^{n}|\lambda|} .
\end{aligned}
$$

Now, we have

$$
\begin{aligned}
& \left\|P_{1, N_{1}}(x)-\bar{u}(x)\right\|_{\infty}=\left\|Q_{1, N_{1}}(x)+\alpha x+\beta-\bar{u}(x)\right\|_{\infty} \\
& \leq\left\|Q_{1, N_{1}}(x)-\bar{u}(x)\right\|_{\infty}+\|\alpha+\beta\|_{\infty} \leq \frac{3 \epsilon}{16 \times 2^{n}|\lambda|}<\frac{\epsilon}{5 \times 2^{n}|\lambda|}, \\
& \left\|\frac{d^{2} P_{1, N_{1}}(x)}{d x^{2}}-\frac{d^{2} \bar{u}(x)}{d x^{2}}\right\|_{\infty}=\left\|\frac{d^{2} Q_{1, N_{1}}(x)}{d x^{2}}-\frac{d^{2} \bar{u}(x)}{d x^{2}}\right\|_{\infty} \leq \frac{\epsilon}{16 \times 2^{n}|\lambda|}<\frac{\epsilon}{5 \times 2^{n}|\lambda|} .
\end{aligned}
$$

Since $C^{2}[0,1]$ is a Banach algebra, we may have

$$
\begin{aligned}
\left\|P_{1, N_{1}}^{2}(x)-\bar{u}^{2}(x)\right\|_{\infty} & \leq\left\|P_{1, N_{1}}(x)-\bar{u}(x)\right\|_{\infty}\left\|P_{1, N_{1}}(x)+\bar{u}(x)\right\|_{\infty} \\
& \leq \frac{\epsilon}{16 \times 2^{n}|\lambda|\left(\left\|Q_{1, N_{1}}(x)\right\|_{\infty}+\|\bar{u}(x)\|_{\infty}\right)}\left(\left\|Q_{1, N_{1}}(x)\right\|_{\infty}+\|\bar{u}(x)\|_{\infty}\right) \\
& <\frac{\epsilon}{5 \times 2^{n}|\lambda|} .
\end{aligned}
$$

Now, we define

$$
\begin{aligned}
L P_{N}(x) & =L\left(P_{1, N_{1}}(x), \frac{d^{2} P_{1, N_{1}}(x)}{d x^{2}}\right) \\
& =\frac{d^{2} P_{1, N_{1}}(x)}{d x^{2}}+\lambda\left(1+P_{1, N_{1}}(x)+\frac{P_{1, N_{1}}^{2}(x)}{2 !}+\frac{P_{1, N_{1}}^{3}(x)}{3 !}+\ldots\right)=F(x)
\end{aligned}
$$

for every $x \in[0,1]$. Thus for $N \geq N_{1}$, we find an upper bound for the following residual:

$$
\begin{aligned}
\left\|L P_{N}(x)-F(x)\right\|_{\infty} & =\left\|L\left(P_{1, N_{1}}(x), \frac{d^{2} P_{1, N_{1}}(x)}{d x^{2}}\right)-F(x)\right\|_{\infty} \\
& \leq\left\|\frac{d^{2} P_{1, N_{1}}(x)}{d x^{2}}-\frac{d \bar{u}^{2}(x)}{d x^{2}}\right\|_{\infty}+|\lambda|\left(\left\|P_{1, N_{1}}(x)-\bar{u}(x)\right\|_{\infty}+\frac{1}{2}\left\|P_{1, N_{1}}^{2}(x)-\bar{u}^{2}(x)\right\|_{\infty}+\ldots\right) \\
& \leq \epsilon .
\end{aligned}
$$

Since the residual $R\left(P_{N}\right):=L P_{N}(x)-F(x)$ is a polynomial, we can represent it by a Bezier form. Thus we have

$$
R\left(P_{N}\right):=\sum_{i=0}^{m} d_{i, m} B_{i, m}(x)
$$

Then from Lemma 1 in [29], there exists an integer $M(\geq N)$ such that when $m>M$, we have

$$
\left|\frac{1}{m+1} \sum_{i=0}^{m} d_{i, m}^{2}-\int_{0}^{1}\left(R\left(P_{N}\right)\right)^{2} d x\right|<\epsilon,
$$

which gives

$$
\frac{1}{m+1} \sum_{i=0}^{m} d_{i, m}^{2}<\epsilon+\int_{0}^{1}\left(R\left(P_{N}\right)\right)^{2} d t \leq \epsilon
$$


Suppose $u(x)$ is a approximated solution of (14) obtained by the control-point-based method of degree $k(k \geq m \geq M)$. Let

$$
R\left(u(x), \frac{d u(x)}{d x}\right)=L\left(u(x), \frac{d u(x)}{d x}\right)-F(x)=\sum_{i=0}^{k} c_{i, k} B_{i, k}(x), \quad k \geq m \geq M, \quad x \in[0,1] .
$$

Define the following norm for difference approximated solutions $u(x)$ and exact solutions $\bar{u}(x)$ :

$$
\|u(x)-\bar{u}(x)\|:=\int_{0}^{1} \sum_{j=0}^{2}\left|\frac{d^{j} u(x)}{d x^{j}}-\frac{d^{j} \bar{u}(x)}{d x^{j}}\right|^{2} d x .
$$

It is easy to show that:

$$
\begin{aligned}
\|u(x)-\bar{u}(x)\| & \leq C\left(|u(0)-\bar{u}(0)|+\left|\frac{d u}{d x}\right|_{x=0}-\left.\frac{d \bar{u}}{d x}\right|_{x=0} \mid+\left\|R\left(u(x), \frac{d u(x)}{d x}\right)\right\|_{2}^{2}\right) \\
& =C \int_{0}^{1} \sum_{i=0}^{k}\left(c_{i, k} B_{i, k}(t)\right)^{2} d x \\
& \leq \frac{C}{k+1} \sum_{i=0}^{k} c_{i, k}^{2} .
\end{aligned}
$$

The last inequality in (18) is obtained from Lemma 1 in [29] in (18) which $C$ is a constant positive number. Now, from Lemma 1 and (16), one can easily show that:

$$
\| u(x))-\bar{u}(x) \| \leq \frac{C}{k+1} \sum_{i=0}^{k} c_{i, k}^{2} \leq \frac{C}{k+1} \sum_{i=0}^{k} d_{i, k}^{2} \leq \frac{C}{m+1} \sum_{i=0}^{m} d_{i, m}^{2} \leq C(\epsilon)=\epsilon_{1}, m \geq M,
$$

where the last inequality in (19) comes from (16).

This completes the proof.

\section{Numerical applications}

To illustrate the ability of this method, some examples are given. The findings reveal that the method is very successful for obtaining a solution of the Bratu's problem.

Example 1. Consider the following Bratu's problem:

$$
\begin{aligned}
& u^{\prime \prime}-2 e^{u}=0,0<x<1, \\
& u(0)=u(1)=0 .
\end{aligned}
$$

The exact solution is $u(x)=-2 \ln (\cos (x))$. We use the proposed method with $n=8$ for solving this problem. The graphs of approximate and exact solutions $u(x)$ are plotted in Figure 1 (with $n=8$ ). Table 1 demonstrates the comparison between the absolute error of the proposed technique, Chebyshev wavelets (with $k=1$ and $M=6$ ) [35], and Legendre wavelets [35]. Table 2 gives the comparision of the error with different $n$ (for the convergence of the present method). The computation takes 3 seconds of CPU time when it is performed using Maple 12 on an AMD Athelon X4 PC with 2 GB of RAM. The NLPSolve command solves (8), which involves computing the minimum of a nonlinear objective function possibly subject to linear constraints. For $n=3$, we have

$$
u_{\text {bezier }}=(1-x)^{3} c_{0}+3 x(1-x)^{2} c_{1}+3 x^{2}(1-x) c_{2}+t^{3} c_{3} .
$$


In Maple with Eq. (20), we have

$$
\begin{aligned}
& f:=1+x+x^{2} / 2+x^{3} / 6 \\
& R 2:=\operatorname{subs}\left(t=u_{\text {bezier }}, f\right) \text {; } \\
& \text { eqq1 := diff }\left(u_{\text {bezier }},[x \$ 2]\right)-2 \star R 2 \text {; } \\
& h:=10 ; a:=0 ; b:=1 \text {; } \\
& \text { for } i \text { from } 0 \text { by } 1 \text { to } h \text { do } \\
& x[i]:=a+((b-a) / h) \star i \text {; } \\
& e q[i]:=\operatorname{subs}(x=x[i], e q q 1) \text {; } \\
& \text { enddo; } \\
& \text { obj :=0; } n:=3 \text {; } \\
& \text { for } i \text { from } 1 \text { by } 1 \text { to } n-1 \text { do } \\
& o b j:=o b j+c[i]^{2} \text {; } \\
& \text { enddo : } \\
& \text { cnsts := [eq[0],eq[1],eq[2],eq[3],eq[4],eq[5],eq[6],eq[7],eq[8],eq[9],eq[10]]; } \\
& \text { NLPSolve(obj, cnsts); }
\end{aligned}
$$

Now, by substititing into Eq. (8), we obtain the following results:

$$
\begin{gathered}
c_{0}=0, \quad c_{1}=-2.71050543121376 \times 10^{-20}, \\
c_{2}=-2.71050543121376 \times 10^{-20}, \quad c_{3}=-2 \ln (\cos (1)), \\
u_{\text {bezier }}=-8.131516294 \times 10^{-20}+8.131516294 \times 10^{-20} x^{2}+1.231252941 x^{3} .
\end{gathered}
$$

If we select $\lambda=3.51$ with $n=8$, the following results are achieved

$$
\begin{aligned}
u_{\text {bezier }}= & 4.19840927 x^{8}-4.293691442 x^{2}+15.95911927 x^{3}-59.2204564 x^{4} \\
& +62.12047483 x^{5}-15.87307814 x^{6}-9.093378061 x^{7}+6.202600671 x .
\end{aligned}
$$

Table 3 gives the error with $n=3.51$.

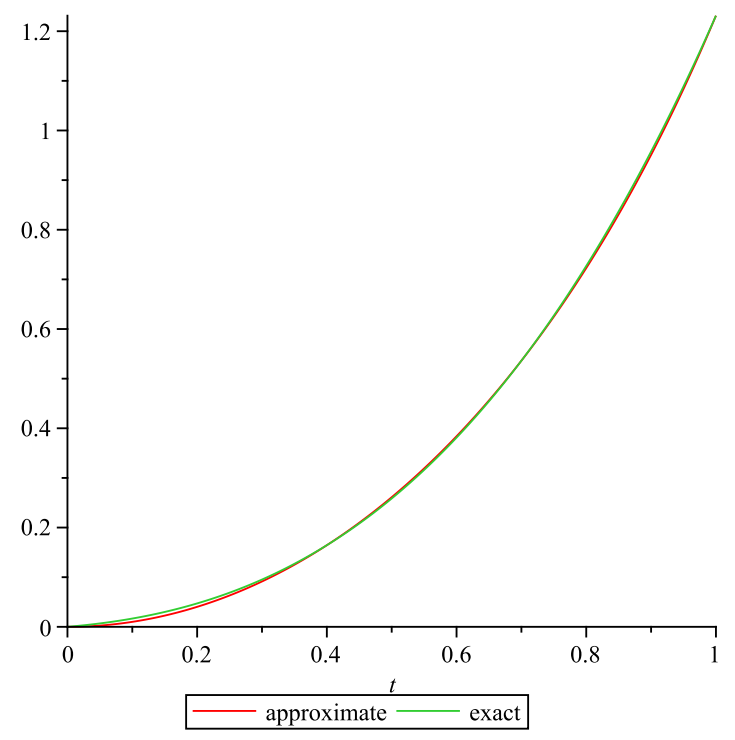

Figure 1: The graphs of approximated and exact solutions $u(x)$ for Example 1. 
Table 1: Comparison of the error for proposed method, Chebyshev, and Legendre wavelets method for Example 1.

\begin{tabular}{llll}
\hline$x$ & error of $u$ for the proposed method & error of Chebyshev wavelets & error of Legendre wavelets \\
\hline 0.1 & $2.529410000 \times 10^{-12}$ & 0.0 & $9 \times 10^{-6}$ \\
0.2 & $2.472000000 \times 10^{-12}$ & $4 \times 10^{-7}$ & $15 \times 10^{-6}$ \\
0.3 & 0.0001918049054 & $15 \times 10^{-7}$ & $0.614 \times 10^{-4}$ \\
0.4 & 0.0001658529272 & $6 \times 10^{-7}$ & $0.888 \times 10^{-4}$ \\
0.5 & $1.000000000 \times 10^{-12}$ & $0.737 \times 10^{-4}$ & $0.5669 \times 10^{-3}$ \\
0.6 & 0.00005651941000 & $0.53673 \times 10^{-2}$ & $0.25577 \times 10^{-2}$ \\
0.7 & $7.000000000 \times 10^{-11}$ & $4 \times 10^{-5}$ & $0.92461 \times 10^{-2}$ \\
0.8 & $1.000000000 \times 10^{-10}$ & $0.6398 \times 10^{-3}$ & $0.286198 \times 10^{-1}$ \\
0.9 & $2.000000000 \times 10^{-10}$ & $0.1528 \times 10^{-3}$ & $0.791248 \times 10^{-1}$ \\
\hline
\end{tabular}

Table 2: Comparison of the error for the proposed method with different $n$ for Example 1.

\begin{tabular}{lllll}
\hline$x$ & error with $n=3$ & error with $n=5$ & error with $n=7$ & error with $n=15$ \\
\hline 0.1 & 0.008785458259 & 0.01710549764 & 0.01770104387 & $1.231252941 \times 10^{-15}$ \\
0.2 & 0.03041952266 & $1.1 \times 10^{-12}$ & $2.360000000 \times 10^{-12}$ & $3.456963600 \times 10^{-13}$ \\
0.3 & 0.05813948250 & 0.02678752885 & 0.008769247952 & 0.002757317303 \\
0.4 & 0.08565785000 & 0.04294036881 & $1.800000000 \times 10^{-11}$ & $5.222200000 \times 10^{-11}$ \\
0.5 & 0.1072618632 & 0.03517577600 & 0.01101646320 & 0.004087227458 \\
0.6 & 0.1179797036 & $2 \times 10^{-11}$ & $2.000000000 \times 10^{-11}$ & $5.5 \times 10^{-11}$ \\
0.7 & 0.1138517564 & 0.05364174050 & 0.04855446610 & 0.006610826229 \\
0.8 & 0.09237998820 & 0.1018934012 & 0.1210180696 & $1.000000000 \times 10^{-11}$ \\
0.9 & 0.05330149330 & 0.1038404221 & 0.1520070074 & 0.0 \\
\hline
\end{tabular}

Table 3: The error for the proposed method with $n=8$ and $\lambda=3.51$ for Example 1 .

\begin{tabular}{ll}
\hline$x$ & error \\
\hline 0.1 & $1.110223025 \times 10^{-16}$ \\
0.2 & $2.220446049 \times 10^{-16}$ \\
0.3 & 0.003880930546 \\
0.4 & 0.003442827953 \\
0.5 & $2.220446049 \times 10^{-16}$ \\
0.6 & 0.0009925212416 \\
0.7 & $2.220446049 \times 10^{-16}$ \\
0.8 & 0.0 \\
0.9 & $2.220446049 \times 10^{-16}$ \\
\hline
\end{tabular}




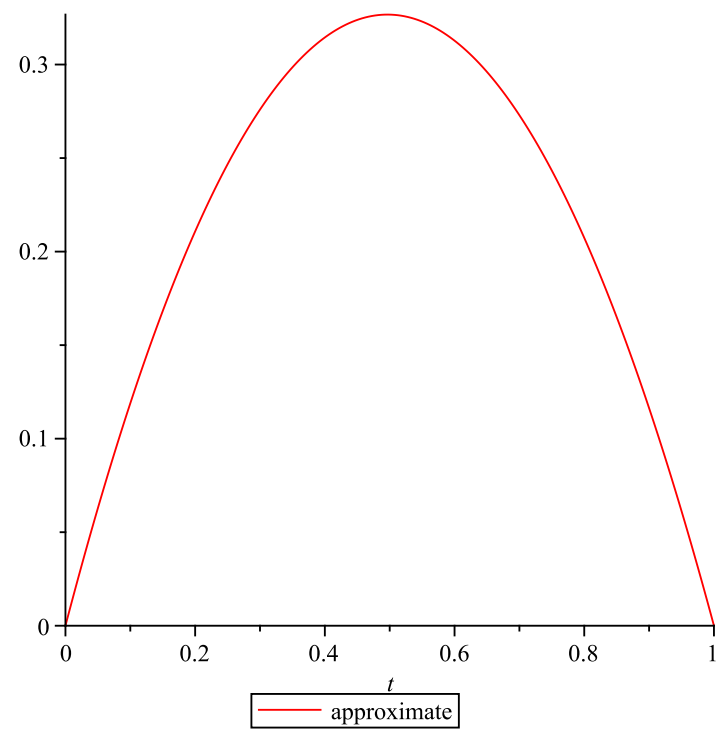

Figure 2: The graph of approximated $u(x)$ for Example 2.

Example 2. Presently, the following problem is considered as follows (see [36]):

$$
\begin{aligned}
& u^{\prime \prime}+2 e^{u}=0, \quad 0<x<1, \\
& u(0)=u(1)=0,
\end{aligned}
$$

These results are obtained by utilizing the Bezier curve technique with $n=8$. The graph of approximated solutions $u(x)$ is plotted in Figure 2. Table 4 demonstrates the comparison between the absolute error of exact and approximated solutions.

Table 4: The exact value and absolute errors of the this method for Example 2.

\begin{tabular}{lll}
\hline$x$ & exact of $u$ & error of $u$ \\
\hline 0.125 & 0.13960278219 & $1.493350000 \times 10^{-11}$ \\
0.250 & 0.24333656779 & 0.0001214358601 \\
0.375 & 0.30731941062 & $4.960000000 \times 10^{-11}$ \\
0.500 & 0.32895242134 & 0.00001053263900 \\
0.625 & 0.30731941062 & $2.000000000 \times 10^{-11}$ \\
0.750 & 0.24333656779 & $1.000000000 \times 10^{-10}$ \\
0.875 & 0.13960278219 & 0.0002111432700 \\
\hline
\end{tabular}

\section{Conclusions}

The aim of this sequel is to improve an effective and accurate technique for solving Bratu's problem. The Bezier curve strategy is utilized to obtain the approximate solution of this problem. The method used in this article reduces the CPU time. Illustrative examples are included to show the validity of this technique. Comparing with other methods, the results of numerical examples demonstrate that this method is more accurate than some existing methods. 
Acknowledgments: The authors wish to acknowledge the editor of this journal. The authors would like to thank the anonymous reviewers of this paper for their careful reading, constructive comments and nice suggestions which have improved the paper very much.

\section{References}

[1] Caglara H., Caglarb N., Özer M., B-spline method for solving Bratu’s problem, Int. J. Comput. Math., 2010, 87(8), 1885-1891

[2] Ascher U. M., Matheij R., Russell R. D., Numerical solution of boundary value problems for ordinary differential equations, SIAM, Philadelphia, 1995

[3] Boyd J. P., Chebyshev polynomial expansions for simultaneous approximation of two branches of a function with application to the one-dimensional Bratu equation, Appl. Math. Comput., 2003, 14, 189-200

[4] Buckmire R., Investigations of nonstandard Mickens-type finite-difference schemes for singular boundary value problems in cylindrical or spherical coordinates, Numer. Methods Partial Differ. Equ., 2003, 19(3), 380-398

[5] Mounim A. S., de Dormale B. M., From the fitting techniques to accurate schemes for the Liouville-Bratu-Gelfand problem, Numer. Methods Partial Differ. Equ., 2006, 22(4), 761-775

[6] Syam M. I., Hamdan A., An efficient method for solving Bratu equations, Appl. Math. Comput., 2006, 176, 704-713

[7] Li S., Liao S. J., Analytic approach to solve multiple solutions of a strongly nonlinear problem, Appl. Math. Comput., 2005, $169,854-865$

[8] Wazwaz A. M., Adomian decomposition method for a reliable treatment of the Bratu-type equations, Appl. Math. Comput., 2005, 166, 652-663

[9] Ali M. R., Hadhoud A. R., Hybrid orthonormal Bernstein and block-pulse functions wavelet scheme for solving the 2D Bratu problem, Results in Physics, 2019, 13, 12-21

[10] Ali M. R., Hadhoud A. R., Srivastava H. M., Solution of fractional Volterra-Fredholm integro-differential equations under mixed boundary conditions by using the HOBW method, Adv. Difference Equ., 2019, Article number: 115

[11] Ali M. R., Hadhoud A. R., Application of Haar wavelet method for solving the nonlinear fuzzy integro-differential equations, J. Comp. Theor. Nanos., 2019, 16(1), 1-18

[12] Ali M. R., Mousa M. M., Ma W. X., Solution of nonlinear Volterra integral equations with weakly singular kernel by using the HOBW method, Adv. Math. Phys., 2019, Article ID 1705651

[13] Alomari A. K., Syam M. I., Al-Jamal M. F., Bataineh A. S., Anakira N. R., Jameel A. F., Modified Legendre operational matrix of differentiation for solving strongly nonlinear dynamical systems, Int. J. Appl. Comput. Math., 2018, 4:117

[14] Bataineh A. S., Bernstein polynomials method and it's error analysis for solving nonlinear problems in the calculus of variations: convergence analysis via residual function, Filomat, 2018, 32(4), 1379-1393

[15] Bataineh A. S., Application of adaptation HAM for nonlinear oscillator typified as a mass attached to a stretched elastic wire, Communications in Mathematics and Applications, 2017, 8(2), 157-165

[16] Bataineh A. S., Al-Omari A. A., Isik O. R., Hashim I., Multistage Bernstein collocation method for solving strongly nonlinear damped systems, J. Vib. Control, 2018, 25(1), 122-131

[17] Bataineh A. S., Hashim I., Isik O. R., Solution of fractional-order differential equations based on the operational matrices of new fractional Bernstein functions MHT Alshbool, Journal of King Saud University-Science, 2017, 29(1), 1-18

[18] Bataineh A., Isik O., Aloushoush N., Shawagfeh N., Bernstein operational matrix with error analysis for solving high order delay differential equations, Int. J. Appl. Comput. Math., 2016, 3(3), 1749-1762

[19] Bataineh A. S., Isik O. R., Hashim I., Bernstein method for the MHD flow and heat transfer of a second grade fluid in a channel with porous wall, Alexandria Engineering Journal, 2016, 55(3), 2149-2156

[20] Ghomanjani F., Shateyi S., Numerical solution for fractional Bratu's initial value problem, Open Physics, 2017, 15(1), 10451048

[21] Bronson R., Costa G. B., Schaum's Outline of Differential Equations, 3rd Edn., McGraw-Hill, New York, 2006

[22] Boyd J. P., One-point pseudo spectral collocation for the one-dimensional Bratu equation, Appl. Math. Comput., 2011, 217(12), 5553-5565

[23] Aksoy Y., Pakdemirli M., New perturbation iteration solutions for Bratu-type equations, Comput. Math. Appl., 2010, 59, 2802-2808

[24] Jalilian R., Non-polynomial spline method for solving Bratu's problem, Comput. Phys. Commun., 2010, 181, 1868-1872

[25] Rashidinia J., Maleknejad K., Taheri N., Sinc-Galerkin method for numerical solution of the Bratu's problem, Numer. Algorithms, 2013, 62, 1-11

[26] Venkatesh S. G., Ayyaswamy S. K., Raja Balachandar S., The Legendre wavelet method for solving initial value problems of Bratu-type, Comput. Math. Appl., 2012, 63(8), 1287-1295

[27] Harada K., Nakamae E., Application of the Bezier curve to data interpolation, Computer-Aided Design, Int. J. Comput. Math., 1982, 14(1), 55-59

[28] Nürnberger G., Zeilfelder F., Developments in bivariate spline interpolation, J. Comput. Appl. Math., 2000, 121(1-2), 125-152 
[29] Zheng J., Sederberg T. W., Johnson R. W., Least squares methods for solving differential equations using Bezier control points, Appl. Numer. Math., 2004, 48, 237-252

[30] Ghomanjani F., Farahi M. H., The Bezier control points method for solving delay differential equation, Intelligent Control and Automation, 2012, 3(2), 188-196

[31] Ghomanjani F., Farahi M. H., Gachpazan M., Bezier control points method to solve constrained quadratic optimal control of time varying linear systems, Comput. Appl. Math., 2012, 31(3), 433-456

[32] Ghomanjani F., Farahi M. H., Optimal control of switched systems based on Bezier control points, Int. J. Intell. Syst. Appl., 2012, 4(7), 16-22

[33] Ghomanjani F., Farahi M. H., Kamyad A. V., Numerical solution of some linear optimal control systems with pantograph delays, IMA J. Math. Control Inform., 2015, 32(2), 225-243

[34] Xie L. J., Zhou C. L., Xu S., An effective numerical method to solve a class of nonlinear singular boundary value problems using improved differential transform method, Springerplus, 2016, 5:1066

[35] Yang C., Hou J., Chebyshev wavelets method for solving Bratu’s problem, Bound. Value Probl., 2013, $2013: 142$

[36] Zarebnia M., Sarvari Z., New approach for numerical solution of the one-dimensional Bratu equation, Thai J. Math., 2013, 11(3), 611-621 